\title{
On the Use of Radiation Technology for Nanoscale Engineering of Silver/ Hydrogel Based Nanocomposites for Potential Biomedical Application
}

\author{
Z. Kačarević-Popović*,, M. Dragašević ${ }^{2}$, A. Krklješ ${ }^{1}$, S. Popović ${ }^{2}, \check{Z}$. Jovanović ${ }^{3}$, S. Tomić ${ }^{3}$ and \\ V. Mišković-Stanković ${ }^{3}$
}

${ }^{1}$ Vinča Institute of Nuclear Science, P.O. Box 522, 11001 Belgrade, Serbia; ${ }^{2}$ Institute of Endocrinology, Diabetes and Metabolic Diseases, University Clinical Centre of Serbia, Dr Subotića 13, 11000 Belgrade, Serbia; ${ }^{3}$ Faculty of Technology and Metallurgy, University of Belgrade, P.O. Box 3503, 11120 Belgrade, Serbia

\begin{abstract}
For nanoscience to become true nanotechnology, there is a need for breakthroughs in the engineering science of processing and manufacturing at the nanoscale. The radiation technology may offer a novel approaches to solving the problems of placement, high throughput, as well as integration across multiple length scales. Furthermore, there are critical needs for advanced materials in the area of biomaterial engineering, primarily in generating biomaterials of enhanced specific functionalities, which can be achieved by introduction of proper functionalities at the nanoscale dimensions. The radiation techniques are uniquely suited for such task, due to their favorable characteristics and in most cases not possible by other methods of synthesis. Therefore, we are systematically developing novel synthetic strategies for incorporation of noble metal nanoparticles in hydrogel networks by gamma irradiation, for possible biomedical application, using liquid filled cavities in hydrogels as nanoreactors (template synthesis). The radiation process has various advantages, such as easy process control, the possibility of joining synthesis and sterilization in one technological step. The radiation technique does not require any extra substances, and does not need any further purification. On the other hand, in recent years nanoscale antibacterial materials, such as nanocrystalline silver, as novel antimicrobial species have been seen as promising candidates for application owing to their high surface to volume ratio and their novel physical and chemical properties on the nanoscale level. Silver can be safely used even for patients who have diseases like Diabetes Mellitus that interfere with wound healing. The recent emergence of nanotechnology has provided a new therapeutic modality in silver nanoparticles for healing wounds.
\end{abstract}

Keywords: Gamma irradiation induced synthesis, Ag/polymer hydrogel nanocomposites, potential biomedical application.

\section{INTRODUCTION}

High energy radiation is capable of producing ionization in substances. Ionizing radiation consists of subatomic particles or electromagnetic waves that are energetic enough to detach electrons from atoms or molecules, ionizing them. Ionizing particles are energetic alpha particles, beta particles, and neutrons. Radiation on the short-wavelength end of the electromagnetic spectrum such as high frequency ultraviolet, $x$-rays, and gamma rays is ionizing. Biological and medical applications of ionizing irradiation includes: medical radiography; radiation therapy; tracer methods; in biology and agriculture to induce mutations in insect control; in medicine, and other fields, radiation is used for sterilization of tools, equipment and food. Moreover, in the area of biomaterial engineering the radiation techniques are uniquely suited for generating biomaterials of enhanced specific functionalities due to their favorable characteristics and in most cases not possible by other methods of synthesis. This can be achieved by introduction of proper functionalities at the nanoscale dimensions using ionizing irradiation.

On the other hand, the number of cases of diabetes worldwide in 2000 among adults, 20 years of age is

*Address correspondence to this author at the Vinča Institute of Nuclear Science, P.O. Box 522, 11001 Belgrade, Serbia; Tel: 381-11-3408357; Fax: 381-11-3408607; E-mail: zkacar@ vinca.rs estimated to be 171 million [1]. These data provide an updated quantification of the growing public health burden of diabetes across the world. The human and economic costs of this epidemic are enormous. This will inevitably result in increased prevalence and associated consequences of complications of diabetes. Among them diabetic foot ulcers (DFU) affect $15 \%$ of diabetic patients and cause longer hospitalization than any other complication of diabetes. Contrary to popular opinion that diabetic foot is caused by neuropathy and peripheral vascular disease, it now appears that disregulated apoptosis is emerging as major cause of the diabetic foot wound [2].

In general, the treatment of diabetic wounds is often more difficult than other chronic wounds:

1. The normal infection reaction would be further disturbed by a poor vascular response.

2. The normal granulocyte migration would be delayed.

3. Chemotaxis (attraction of cells by chemical substances) would develop less effectively in case of hyperglycemia.

4. The same hyperglycemia would reduce the granulocytes phagocytosis capacity. 


\section{Wound Dressings}

Wound dressings represent a part of the management of diabetic foot ulcerations. Ideally, dressings should alleviate symptoms, provide wound protection, and encourage healing. No single dressing fulfils all the requirement of diabetic patient with an infected foot ulcer. Dressings research in this area is generally poor. However each category of dressings has particular characteristics that aid selection. A simple rule may be followed when selecting the appropriate dressing for diabetic plantar foot ulcers. High levels of exudate warrant the choice of a moisture-absorbing material, which may include alginates, foams, collagenalginate combinations, carboxymethylcellulose materials, or gauze. Low exudate and desiccated wounds respond well to hydrogels. Nonadhesive dressings are simple, inexpensive and well tolerated. Foam and alginate dressings are highly absorbent and effective for heavily exuding wounds. Dressings containing iodine and silver may aid in managing wound infection. Hydrogels facilitate autolysis and may be beneficial in managing ulcers containing necrotic tissue [3]. Autolysis is most effective in a moist environment. Hydrogels have the ability to donate water molecules to dehydrated tissue while allowing the passage of water vapor and oxygen to the wound surface [4]. This helps to increase the phagocytic activity of leucocytes and enzymatic activity of damaged cells. This in turn removes devitalised tissue during the destructive phase of healing of wound-autolysis [5]. But in general, the fluid handling properties of hydrogels are different. Some of them are donators of fluid and more suited for use with dry, necrotic wounds to promote hydration and autolysis. Some of them have both fluid donation and absorption capabilities. Finally some of them have better fluid absorbing properties indicating a clinical use more suitable for treating slough and necrotic wounds. Hydrogels could also act as carriers for growth factors, biologically active molecules and antimicrobial agents.

Patients with diabetes are more prone to infection, as they have reduced leukocyte infiltration, decreased phagocytosis, poor bactericidal activity and build-up of debris. Therefore hydrogels should be used with caution in treating infected wounds, as their application may increase the risk of organism spreading to the surrounding tissue [4]. Most of the infections in diabetic wounds are polymicrobal. Silver has been used in the clinical settings as an antimicrobial for over a century, and silver nitrate is still a common antimicrobial used in the treatment of chronic wounds [2]. Silver nitrate causes a significant amount of staining of virtually any surface with which it comes into contact and can also cause irritation of tissues. Silver sulfadiazine was introduced in 1960s to overcome some of the shortcomings of silver nitrate, but both are limited in the clinic due to the need for a high frequency of application, inactivation of much of the silver by wound fluid, and formation of pseudo-eschar. New silver-impregnated dressings were designed to overcome these limitations, in particular the rapid inactivation of silver. In these dressings, as silver is consumed by interaction with target cells or inactivated by protein and anion complexes in wound fluid, additional silver is released, thus producing a sustained, steady supply of active silver.

The in vitro study has shown that the prevalence of silver resistance genes in bacteria isolated from diabetic foot ulcers is low. Using polymerase chain reaction to screen for three silver-resistance transcriptional units-silE, silS and silP-two silver resistant bacteria were identified, both are strains of an enteric bacterium, Enterobacter cloacae, which is not a primary wound pathogen. No recognized wound pathogens (Staphylococcus aureus-24 isolates and Pseudomonas aeruginosa-nine isolates) were found to contain silverresistant genes. The study also shown that low proportion of wound bacteria that possess silver resistance genes are killed when challenged with silver containing wound dressing [6]. Results suggest that presence of silver resistance genes are rare and that genetic resistance does not necessary translate to phenotypic resistance to silver. The treat of widespread resistance is low and therefore silver-containing dressings remain an extremely important tool in managing wound infections.

In Fig. (1) treatment of diabetic foot wound with the silver containing topical dressing at the Institute of Endocrinology, Diabetes and Metabolic Diseases, University Clinical Centre of Serbia, Belgrade is shown. The medical management of diabetes is clinically demanding; typically, a holistic approach is adopted. A key challenge in this therapy area is to prevent and manage the onset of foot problems and ulceration. If foot ulceration occurs, current practices to treat the ulcer include debridement, dressing the wound and pressure relief. An important additional consideration is the active prevention and treatment of infection as this will help contribute to wound closure over a shorter time period than might otherwise be expected. An ideal $\mathrm{Ag}^{+}$donor site dressing material would promote healing, cause minimal pain to the patient, prevent infection, result in minimal scarring, and be inexpensive and easy to use. A dressing which possesses all of these qualities has yet to be developed, but currently many dressing materials meet some of these criteria to varying degrees.

\section{Nano Silver in Biomedical Application}

In recent years nanoscale antibacterial materials as novel antimicrobial species have been seen as promising candidates for application owing to their high surface to


Fig. (1). Treatment of diabetic foot wound with the silver containing topical dressing at the Institute of Endocrinology, Diabetes and Metabolic Diseases, University Clinical Centre of Serbia, Belgrade. 
volume ratio and their novel physical and chemical properties on the nanoscale level. Many kinds of nanometersized antibacterial materials such as $\mathrm{TiO}_{2}, \mathrm{ZnO}$, and $\mathrm{MgO}$, chitosan, calamine, copper and silver have been reported on in this area. Among them nanocrystalline silver has been proved to be most effective antimicrobial agent, since silver and its compounds have powerful antimicrobial capability and broad inhibitory biocidal spectra for microbes, including bacteria, viruses and eukaryotic microorganism. Enhanced antibacterial properties of nanocrystalline silver have been demonstrated both in vitro and in vivo. Silver nanoparticles (AgNPs) show excellent antimicrobial activity by binding both to microbial DNA causing loss of its replication ability, preventing bacterial replication and to the sulfhydryl groups of the metabolic enzymes of the bacterial electron transport chain causing their inactivation [7]. Higher concentrations of $\mathrm{Ag}^{+}$ions have been shown to interact with cytoplasmic components and nucleic acids. In addition, micromolar levels of $\mathrm{Ag}^{+}$ions are known to uncouple respiratory electron transport from oxidative phosphorylation [8]. Moreover, metal nanoparticles such as silver provide interesting research area because they have a close lying conduction and valence bands in which electrons move freely. Due to their ability to absorb and scatter the light and biocompatible nature, they are therefore well suited for nanoparticles optical and biological application. Furthermore, in 2005 investigators first reported that AgNPs prevent HIV-1 infection. Findings suggest that the particles interacted with the viral particles in a size-dependent manner, and behaved as lectins-proteins that interact predominantly with glycosylated loci-probably due to the preferential binding of the nanoparticles to the cysteine-rich moieties of the gp120 glycoprotein present in the viral envelope. The interaction of Ag nanoparticles and gp120 prevented viral fusion with cells, therefore preventing infection [9]. In addition to the antimicrobial properties, silver also appears to have anti-inflammatory properties, as suggested by the loss of rubor in chronic wounds [10]. Silver in the form of nanoparticles are effective at decreasing inflammation in postoperative peritoneal adhesion without significant toxic effects. Resent results confirmed that AgNPs can increase the rate of wound closure. On one hand this was achieved through the promotion of proliferation and migration of keratinocites. On the other hand, AgNPs can drive the differentiation of fibroblasts into myofibroblasts, thereby promoting wound contraction [11].

\section{Radiation Technology}

Since the pioneering work of Wichterle and Lim in 1960 on crosslinked HEMA hydrogels [12], hydrogels have been of great interest to biomaterial scientists. Irradiation has been recognized as highly suitable tool to aid in the formation of hydrogels. The radiation process has various advantages, such as easy process control, the possibility of joining hydrogel formation and sterilization in one technological step, the lack of necessity for initiators and crosslinkers, which are possibly harmful and difficult to remove. The radiation technique is clean, because it does not require any extra substances, does not leave some unwanted residues, and does not need any further purification. These qualities make irradiation the method of choice in the synthesis of hydrogels. Moreover, since performances of $A g$ nanoparticles have a considerable association with the structure, surface modality, size and size distribution, a strict control is needed. It has been shown that the morphology, particle size distribution, stability and properties of $\mathrm{Ag}$ nanoparticles are strongly dependent on the method of preparation and specific experimental conditions. Most of the technologies used to incorporate $\mathrm{Ag}$ into polymeric matrixes involve either chemical workups such as reduction, or synthesis of complex Ag compounds, mixing performed Ag particles with polymers or complicated physical techniques, such as sputtering, and plasma deposition. The radiolytic method is also, like for synthesis of hydrogels, very suitable for generating metal particles in solution. This is achieved by radiolytically generated species, solvated electrons and secondary radicals that exhibit strong reduction potentials, and consequently metal ions are reduced at each encounter. The control of particle size is achieved by the use of capping agents such as polymers, which are present during the formation of metal clusters. Polymer molecules interact with the growing metal particles, inhibiting the aggregation process [13].

\section{The Aim of the Work}

The aim of the work is systematically developing synthetic strategies for incorporation of nano-Ag in hydrogel networks by gamma irradiation, using liquid filled cavities in hydrogels as nanoreactors (template synthesis), and exploring favourable characteristics of radiation technology for nanoscale engineering of materials especially for biomedical application such as easy process control and the possibility of joining synthesis and sterilization in one technological step. The radiation technique does not require any extra substances, and does not need any further purification. The incorporation of template technologies with the synthesis techniques such as gamma ray irradiation methods is expected to produce more elaborated AgNPs suitable for biomedical applications.

\section{EXPERIMENTAL}

\section{Synthesis of Nanocomposites}

The processes of hydrogel formation are essentially due to monomer polymerization or crosslinking of preformed polymers. A great variety of methods to promote crosslinking have indeed been used to prepare hydrogels including chemical and physical methods. Among these, high-energy radiation, in particular gamma rays and electron beam, can be used to polymerize unsaturated compounds. This means that water-soluble polymers can be converted into hydrogels using high-energy radiation. Rosiak and coworkers have presented a successful methodology of hydrogel dressing production [12], based on high-energy radiation. These hydrogels are prepared through irradiation of poly(N-vinyl-2-pyrrolidone) (PVP) aqueous solutions. Hydroxyl radicals formed during water radiolysis react with PVP generating macroradicals. One of the resulting reactions from these radicals is recombination leading to crosslinks.

Reactants used in the synthesis of the investigated systems in this work, and their general chemical structure are shown in Fig. (2). The hydrogels were prepared by gamma irradiation radical copolymerization or crosslinking. The reaction mixture was degassed prior to polymerization and 
<smiles>CCCCN1CCCC1=O</smiles>

poly(N-vinyl-2-pyrrolidone)<smiles></smiles>

bisomers $\left(\mathrm{R}=\mathrm{H}\right.$ or $\mathrm{CH}_{3} ; \mathrm{R}_{1}=$ ethylene or propylene groups)

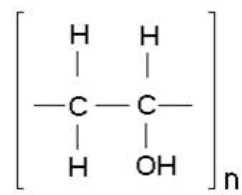

poly(vinyl alcohol)<smiles>C=C(CC(=O)O)C(=O)O</smiles>

itaconic acid

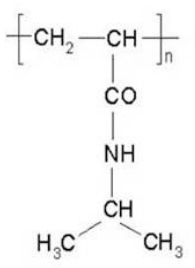

poly(N-isopropylacrylamide)<smiles>C=C(C)C(=O)OCCO</smiles>

2-hydroxyethyl methacrylate

Fig. (2). Reactants used in the synthesis of the investigated systems.

placed between two glass plates, sealed with a spacer. The solutions were irradiated in a ${ }^{60} \mathrm{Co}$ radiation source, under ambient conditions, to absorbed dose of $25 \mathrm{kGy}$. In order to remove any unreacted chemicals, the hydrogels were immersed in deionized water, which was changed every day, for 1 week.

Ag/hydrogel nanocomposites were prepared by swelling the crosslinked polymer samples with water solutions of $\mathrm{AgNO}_{3}$ and propan-2-ol for $48 \mathrm{~h}$. Swelling of Ar-saturated gels was carried out in tightly closed containers at room temperature in the dark. In the second step $\mathrm{Ag}^{+}$ions were reduced in hydrogel using electron transfer reactions from radical species formed in water radiolysis. Gamma irradiation was performed in ${ }^{60} \mathrm{Co}$ radiation facility, at room temperature until achieving complete reduction of $\mathrm{Ag}^{+}$ions.

\section{Characterization of Nanocomposites}

Absorption spectra of Ag/hydrogel nanocomposites were recorded in the wavelength range from 300 to $800 \mathrm{~nm}$ using Thermo Scientific Evolution 600 spectrophotometer.

Transmission electron microscopy (TEM) measurements were performed using Philips EM 400 microscope operated at an accelerating voltage of $120 \mathrm{kV}$.

The X-ray diffraction (XRD) measurements of the $\mathrm{Ag} /$ hydrogel nanocomposites were performed on Bruker D8 Advance Diffractometer $\left(\mathrm{Cu} \mathrm{K}_{\alpha 1}\right.$ radiation, $\left.\lambda=0.1541 \mathrm{~nm}\right)$.

For the swelling studies, the xerogel discs were immersed in an excess of Kokubo's Simulated Body Fluid (SBF) solution [14-16], with the $\mathrm{pH}$ value of 7.1, to obtain equilibrium swelling at $37{ }^{\circ} \mathrm{C}$. SBF is an acellular simulated body fluid that has inorganic ion concentrations similar to those of human extracellular fluid. The simulated body fluid is often abbreviated as SBF or Kokubo solution.

The release of $\mathrm{Ag}^{+}$from $\mathrm{Ag} /$ hydrogel nanocomposites was determined by atomic absorption spectroscopy, by Philips-Pyu Unicam SP 9 AAS spectrophotometer.

Investigation of biological potential of synthesized systems included chemo- and cyto-compatibility.

\section{RESULTS AND DISCUSSION}

The primary products of water radiolysis are shown in Eq. (1):

$$
\mathrm{H}_{2} \mathrm{O} \stackrel{\gamma}{\longrightarrow} \mathrm{e}_{\text {aq }}^{-}(2.7), \mathrm{OH}^{\bullet}(2.7), \mathrm{H}^{\bullet}(0.6), \mathrm{H}_{2}(0.45), \mathrm{H}_{2} \mathrm{O}_{2}(0.7) \text {. }
$$

The numbers in parentheses represents the respective $G$ values. The $G$ value for a given irradiated system is the absolute chemical yield expressed as the number of individual chemical events occurring per $100 \mathrm{eV}$ of absorbed energy [17]. Thus the $\mathrm{G}\left(\mathrm{e}_{\mathrm{aq}}{ }^{-}\right), \mathrm{G}\left(\mathrm{OH}^{\circ}\right)$, etc. are the number of solvated electrons, hydroxyl radicals, etc., formed per 100 $\mathrm{eV}$ of absorbed energy. In the presence of alcohol, the $\mathrm{OH}^{\circ}$ and $\mathrm{H}^{*}$ radicals abstract hydrogen from the alcohol to produce an alcohol radical [18]. It is well known that the radiation crosslinking of polymer (POLYM) molecules is mainly induced by $\mathrm{OH}^{*}$ radicals in aqueous medium (with the $\mathrm{G}$ value of irradiation-induced intermolecular crosslinking 0.48 [19]).

$$
\begin{aligned}
& 2 \mathrm{POLYM}(\mathrm{H})+2 \mathrm{OH}^{\bullet} \rightarrow \text { POLYM }- \text { POLYM } \\
& \text { (crosslinked polymer })+2 \mathrm{H}_{2} \mathrm{O}
\end{aligned}
$$

Under the given experimental conditions $[20,21]$ the $\mathrm{Ag}^{+}$ ions are reduced with strongly reducing hydrated electrons, propan-2-ol radical and the polymeric radicals formed by $\mathrm{H}$ atom abstraction from polymer chains by hydroxyl radicals:

$\mathrm{n} \mathrm{Ag}^{+}+\mathrm{ne}_{\mathrm{aq}}^{-} /\left(\mathrm{CH}_{3}\right)_{2} \mathrm{C}^{\bullet} \mathrm{OH} / \mathrm{POLYM}^{\bullet} \rightarrow(\mathrm{Ag})_{\mathrm{n}}$.

After irradiation, for all compositions, a yellow colored gel was obtained (Fig. (3)).

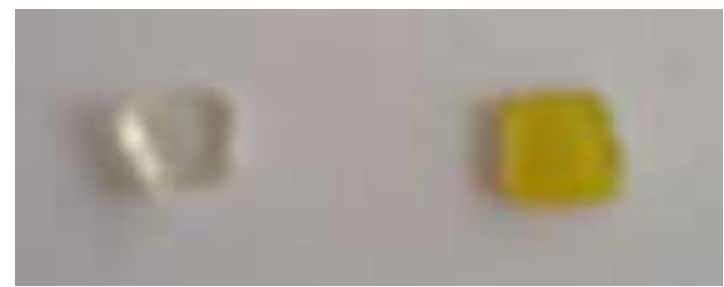

Fig. (3). Photograph of PVP hydrogel (left) and Ag/PVP hydrogel nanocomposite (right). 

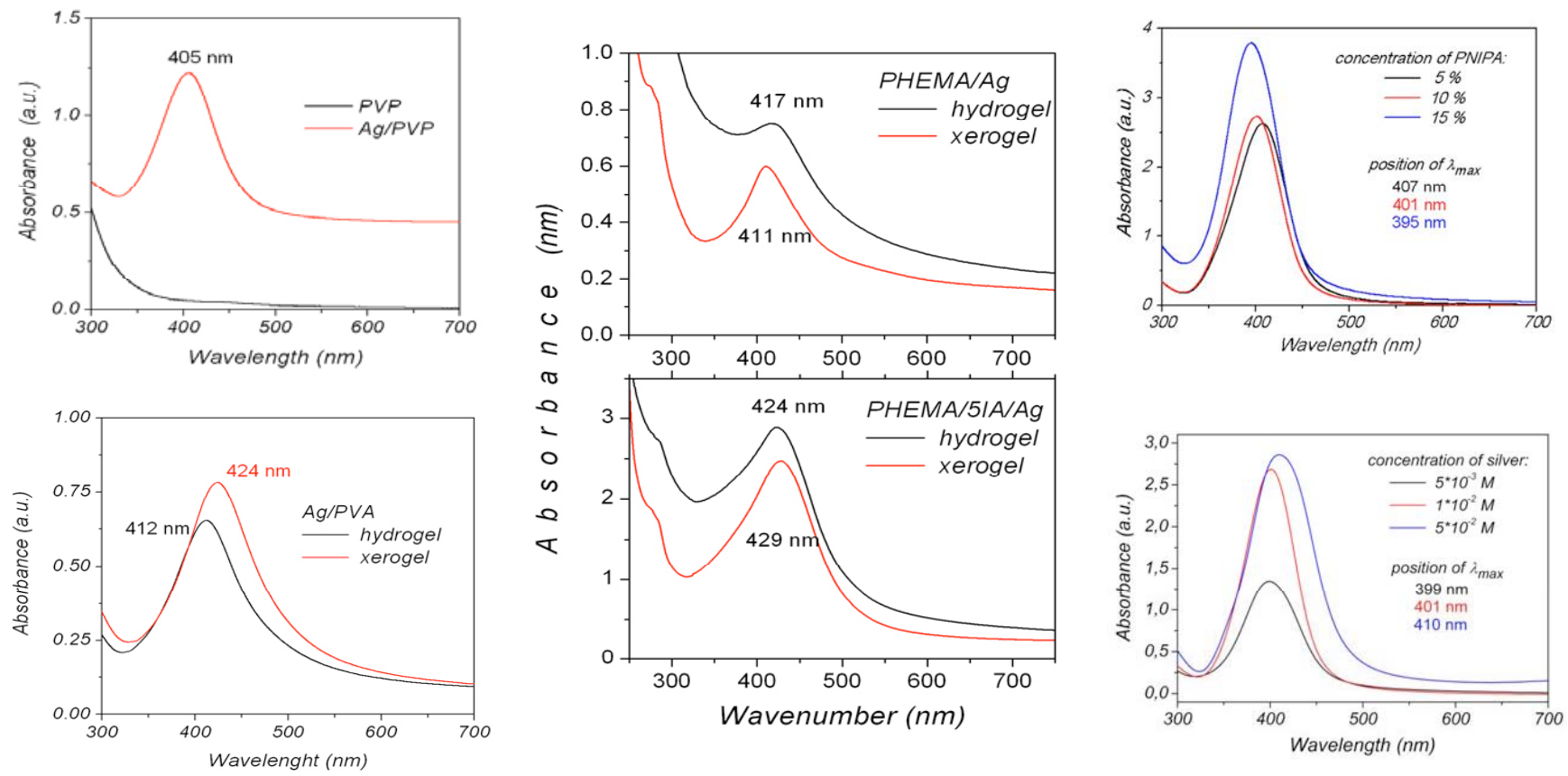

Fig. (4). The appearence of the crystallite surface plasmon in the investigated nanocomposite systems.

Reduction of $\mathrm{Ag}^{+}$ions in hydrogel matrix yielded the typical surface plasmon resonance of $\mathrm{Ag}$ particles for all investigated systems, with no broad absorptions at longer wavelengths, as shown in Fig. (4). It is clearly evident the appearance of the crystallite surface plasmon in different types of investigated nanocomposite systems. Irradiation of $\mathrm{AgNO}_{3}$-loaded hydrogels resulted in a strong sharp absorption centered at $400-430 \mathrm{~nm}$. No significant change in the UV-vis characteristics of the Ag nanoparticles formed in these hydrogels with compositions was observed, as shown in Fig. (4). The sharp absorption pattern indicates that the particle size distribution is quite narrow.

The absorption pattern of the xerogel of the same samples, obtained by the evaporation of the water under vacuum, showed the sharp absorption centered at the same wavelength range. In the dry polymer matrix (xerogel) of all polymer compositions, the signal not only increased in intensity compared to the hydrogel, but also shifted as a consequence of change in dielectric properties of surrounding environment [21].

To probe into the morphologies and size of $\mathrm{Ag}$ nanoparticles, TEM micrographs of $\mathrm{Ag}$ nanoparticles are shown in Fig. (5). It is obvious that these Ag nanoparticles like in all investigated systems assume spherical-like morphologies in appearance at nanoscale levels.

The XRD paterns of PVP and Ag/PVP nanocomposite, are presented in Fig. (6). The XRD peaks, like in all investigated systems exactly matched 111, 200 and 220 crystal planes of Ag with face centered cubic $(f c c)$ crystal structure. The Sherrer diffraction formula was used to estimate the crystalline domain size (d):

$\mathrm{d}=\frac{\mathrm{k} \lambda}{\beta \cos \theta}$

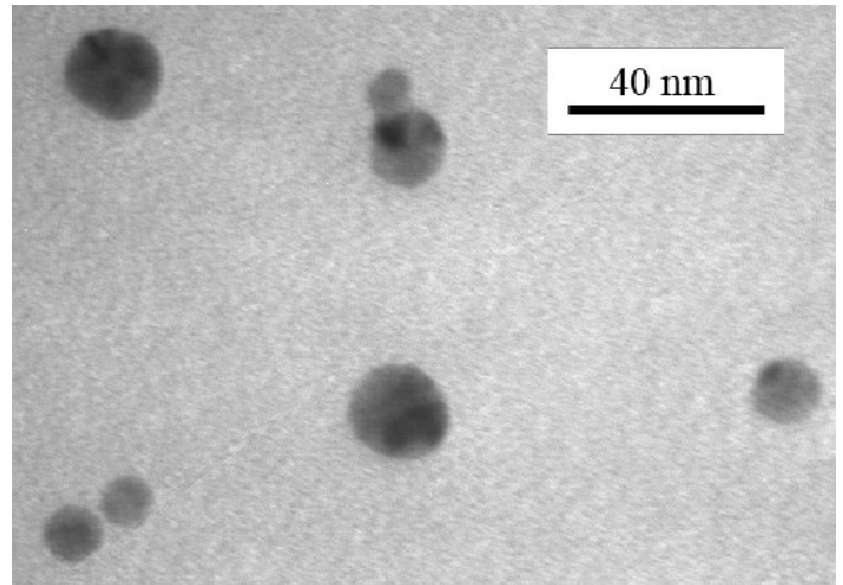

Fig. (5). TEM images of AgNPs formed in PNIPA hydrogel.

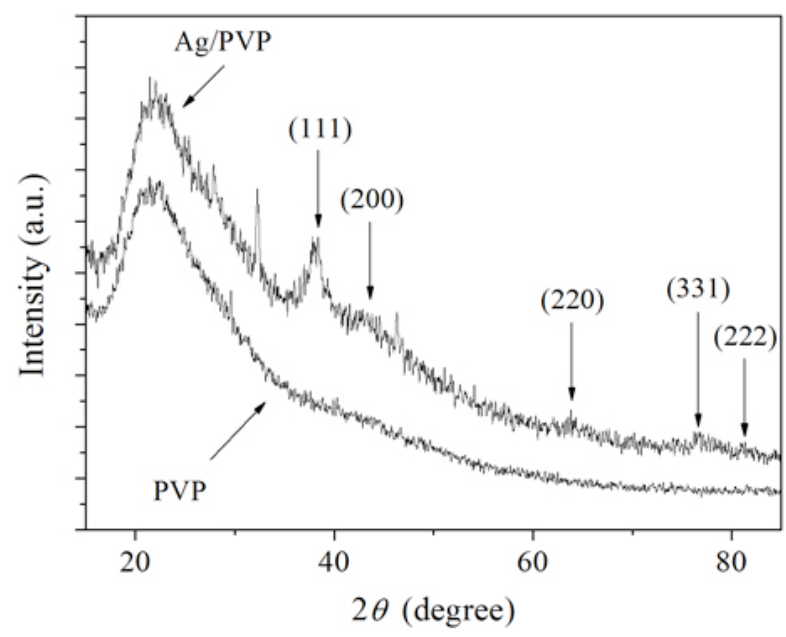

Fig. (6). The XRD patterns of PVP and Ag/PVP nanocomposite. 


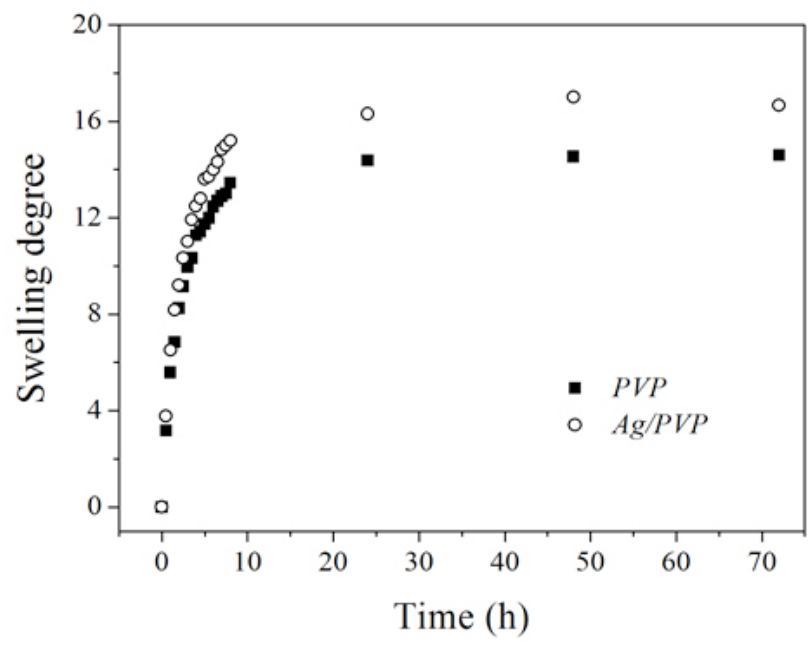

Fig. (7). Swelling curves of PVP and Ag/PVP nanocomposite in SBF solution.
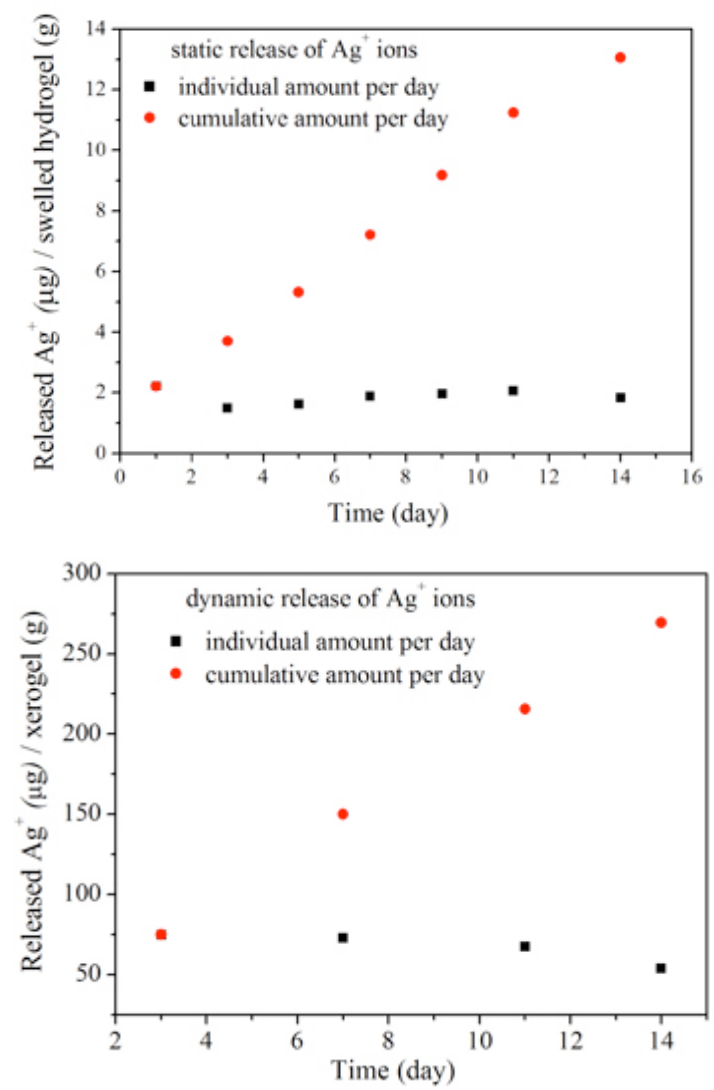

Fig. (8). Release of silver from Ag/PVP hydrogel nanocomposites.

where $\mathrm{k}=0.9$ is constant for the cubic structure, $\lambda=0.1541$ $\mathrm{nm}$ is the X-ray wavelength, $\beta$ is the peak angular width and $\theta$ is the diffraction angle. The crystalline domain size for all systems was found to be about 5-10 nm.

Swelling of the crosslinked polymers in chosen solvent is the most important parameter for swelling studies. Moreover, preliminary studies in buffered solution of $\mathrm{pH}$ similar to the $\mathrm{pH}$ of biological fluids are very important for the application of hydrogels as biomaterials. In general, a fundamental relationship exists between the swelling of a crosslinked polymer and solvent. The intake of xerogels, and $\mathrm{Ag} / \mathrm{xerogel}$ nanocomposite, were followed for a long period of time in $\mathrm{SBF}$ solution at $37{ }^{\circ} \mathrm{C}$. Example of plotted swelling curves is presented in Fig. (7).
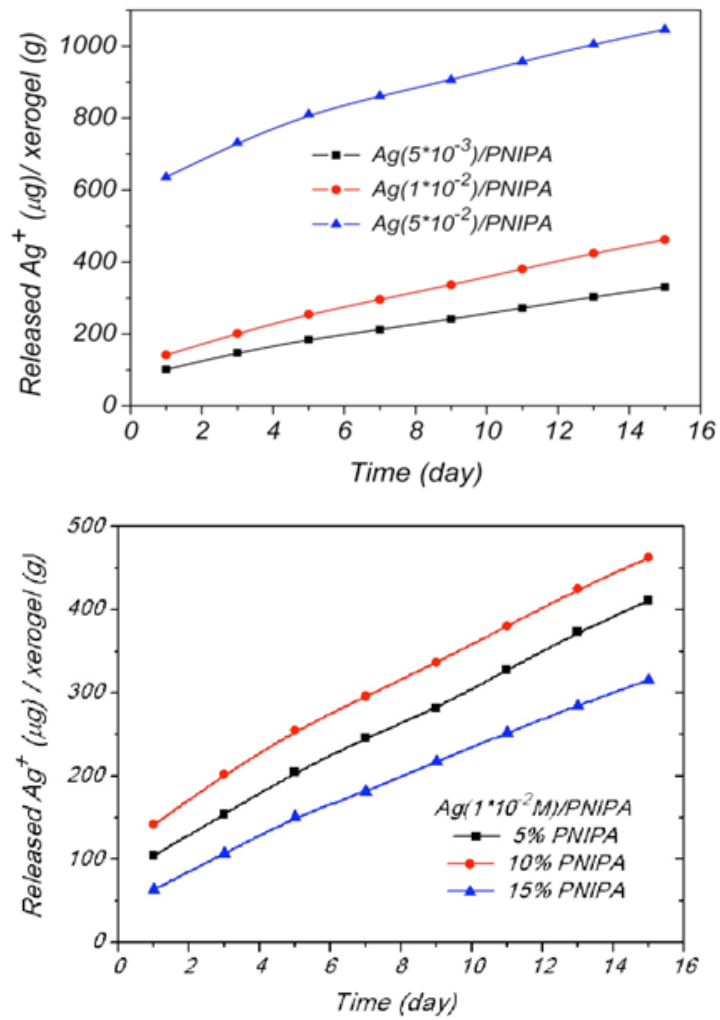

Fig. (9). Release of silver from Ag/PNIPA hydrogel nanocomposites.

As can be seen from Fig. (7), swelling capabilities of PVP hydrogels (xerogel) and Ag/PVP hydrogel (xerogel) nanocomposite are increased by time, reaching constant swelling (equilibrium swelling) after a certain period of time. Another observation is that the swelling of the gels is slightly greater for the gel containing Ag nanoparticles, like in all investigated systems.

Sustained, steady supply of active silver is important property of dressing material. From Figs. (8 and 9) it can be seen that the release of silver $\left(\mathrm{Ag}^{+}\right)$from nanocomposite systems is continious during long period of time which means that investigated hydrogel nanosystems meet that criteria.

The in vitro study of neat hydrogels biocompatibility showed neither evidence of cell toxicity nor any considerable hemolytic activity [22]. Moreover, incorporation of itaconic acid even increased cytocompatibility of neat hydrogels. Furthermore, the microbe penetration test showed that neither Staphylococus aureus nor Escherichia coli passed through the neat hydrogel dressing.

\section{CONCLUSION}

Obtained results indicated that gamma irradiation is suitable for in situ generation of $\mathrm{Ag}$ nanoparticles in investigated hydrogel matrix by radiolytic products of water. $\mathrm{X}$-ray diffraction analysis confirms the $f c c$ structure of $\mathrm{Ag}$ nanoparticles. Swelling properties of synthesized hydrogels, 
neat and $\mathrm{Ag} / \mathrm{hydrogel}$ nanocomposites, investigated in the SBF (simulated body fluid) solution at $37{ }^{\circ} \mathrm{C}$ exhibits that $\mathrm{Ag} /$ hydrogel nanocomposite systems have higher equilibrium swelling compared with neat hydrogel. The release of silver $\mathrm{Ag}^{+}$from nanocomposite systems is continuous during long period of time which means that investigated hydrogel nanosystems meet criteria of sustained, steady supply of active silver.

\section{ACKNOWLEDGEMENTS}

This work is financed by the International Atomic Energy Agency, Vienna, project CRP: F23028 contract No. 15384; Ministry of the Science and Technological development, Republic of Serbia (Contract Nos. 19027 and 142061).

\section{REFERENCES}

[1] Wild, S.; Roglic, G.; Green, A.; Sicree, R.; King, H. Global Prevalence of Diabetes: Estimates for the year 2000 and projections for 2030. Diabetes Care, 2004, 27, 1047-53.

[2] Mishra, M.; Kumar, H.; Tripathi, K. Diabetic delayed woound healing and the role of silver nanoparticles. Dig. J. Nanomater. Biostruct., 2008, 3, 49-54.

[3] Hilton, J.R.; Wiliams, D.T.; Beuker, B.; Miller, D.R.; Harding, K.G. Wound dressings in diabetic foot disease. Clin. Infect. Dis., 2004, 39, 100-3.

[4] Jones, V.; Milton, T. When and How to Use Hydrogels. NT Plus Nursing Times, 2000, 96, 3-4.

[5] Pudner, R. Hydrocolloid dressings in wound management; Alginate and hydrofibre dressings in wound management. J. Commun. Nurs., 2001, 15, 44-52.

[6] Percival, S.L.; Woods, E.; Nutekpor, M.; Bowler, P.; Radford, A.; Cochrane, C. Prevalence of silver resistance in bacteria isolated from diabetic foot ulcers and efficacy of silver-containing wound dressings. Ostomy Wound Manag., 2008, 54, 30-40.

[7] Thomas, V.; Yallapu, M.M.; Sreedhar, B.; Bajpai, S.K. A versatile strategy to fabricate hydrogel-silver nanocomposites and investigation of their antimicrobial activity. J. Coll. Interf. Sci., 2007, 315, 389-95.

[8] Kim J.S. Antibacterial activity of $\mathrm{Ag}^{+}$ion-containing silver nanoparticles prepared using the alcohol reduction method. J. Ind. Eng. Chem., 2007, 13, 718-22.
[9] Elechiguerra, J.L.; Burt, J.L.; .Morones, J.R.; Camacho-Bragado, A.; Gao, X.; Lara, H.H.; Yacaman, M.J. Interaction of silver nanoparticles with HIV-1. J Nanobiotechnol., 2005, 3, 6.

[10] Wong, K.K.Y.; Cheung, S.O.F.; Huang, L.; Niu, J.; Tao, C.; Ho, C.M.; Che, C.M; Tam, P.K.H. Further Evidence of the Antiinflammatory Effects of Silver Nanoparticles. ChemMedChem. 2009, 4, 1129-35.

[11] Liu, X.; Lee, P.Y.; Ho, C.M.; Lui, V.C.H.; Chen, Y.; Che, C.M.; Tam, P.K.H.; Wong, K.K.Y. Silver Nanoparticles Mediate Differential Responses in Keratinocytes and Fibroblasts during Skin Wound Healing. Chem Med Chem. 2010, 5, 468-75.

[12] Fechine, G.J.M.; Barros, J.A.G.; Catalani, L.H. Poly(N-vinyl-2pyrrolidone) hydrogel production by ultraviolet radiation: new methodologies to accelerate crosslinking. Polymer, 2004, 45, 470509.

[13] Krklješ, A.; Marinović-Cincovoć, M.; Kačarević-Popović. Z.; Nedeljković, J. Radiolytic synthesis and characterization of AgPVA nanocomposites. Eur. Polym. J., 2007, 43, 2171-76.

[14] Kokubo, T.; Kushitani, H.; Sakka, S.; Kitsugi, T.; Yamamuro, T. Solutions able to reproduce in vivo surface-structure changes in bioactive glass-ceramic A-W ${ }^{3}$. J. Biomed. Mater. Res., 1990, 24, 721-34.

[15] Bell, C.L.; Peppas, N.A. Measurement of the swelling force in ionic polymer networks. III. Swelling force of interpolymer complexes. J. Control. Release, 1995, 37, 277-80.

[16] Peppas, N.A. Analysis of Fickian and non-Fickian drug release from polymers. Pharm. Acta. Helv., 1985, 60, 110-11.

[17] Draganic, I.G., Draganic Z.D. The Radiation Chemistry of Water; Academic Press: New York, 1971

[18] Kumar, M.; Varshney, L.; Francis, S. Radiolytic formation of Ag clusters in aqueous polyvinyl alcohol solution and hydrogel matrix. Radiat. Phys. Chem., 2005, 73, 21-7.

[19] Wang, B.; Kodama, M.; Mukataka, S.; Kokufuta, E. On the intermolecular crosslinking of PVA chains in an aqueous solution by $\gamma$-ray irradiation. Polym. Gels. Netw., 1998, 6, 71-81.

[20] Kačarević-Popović, Z.; Tomić, S.; Krklješ, A.; Mićić, M.; Suljovrujić, E. Radiolytic synthesis of Ag-poly(BIS-co-HEMA-coIA) nanocomposites. Radiat. Phys. Chem., 2007, 76, 1333 -6.

[21] Krklješ, A.; Nedeljković, J.; Kačarević-Popović, Z. Fabrication of Ag-PVA hydrogel nanocomposite by $\gamma$-irradiation. Polym. Bull., 2007, 58(1), 271-9.

[22] Tomić, S.Lj.; Mićić, M.M.; Krezović, B.D.; Dobić, S.N.; Suljovrujić, E.H.; Filipović, J.M. Smart hydrogels based on itaconic acid for biomedical application. Hem. Ind., 2009, 63, 60310.

(C) Kačarević-Popović et al.; Licensee Bentham Open.

This is an open access article licensed under the terms of the Creative Commons Attribution Non-Commercial License (http://creativecommons.org/licenses/by-nc/3.0/) which permits unrestricted, non-commercial use, distribution and reproduction in any medium, provided the work is properly cited. 